\title{
Lateral Sinus Augmentation: A Safer Technique
}

\author{
Gregori M Kurtzman, Douglas F Dompkowski
}

\begin{abstract}
The posterior maxilla presents with a common problem clinically following tooth extraction or crestal bone loss resulting in loss of osseous height sufficient to place implants. Resorptive patterns in some patients along with sinus enlargement result in minimal bone that can accommodate implant placement. Maxillary sinus augmentation over the past 18 years with various bone graft materials has become routine treatment. Numerous studies have reported highly successful implant survival rates when placed into the augmented sinus. The most common complication of the lateral sinus elevation approach is typically tearing of the Schneiderian membrane which could allow for bacterial contamination or loose particles to gain access to the sinus cavity. A safer lateral window approach sinus augmentation procedure will be discussed using specialized safe cutting end drills with vertical stoppers for osseous window formation and subsequent membrane elevation.
\end{abstract}

Keywords: Sinus augmentation, Lateral sinus approach, Implant.

How to cite this article: Kurtzman GM, Dompkowski DF. Lateral Sinus Augmentation: A Safer Technique. Int J Oral Implantol Clin Res 2013;4(3):122-128.

Source of support: Nil

Conflict of interest: None

\section{INTRODUCTION}

The posterior maxilla presents with a common problem clinically following tooth extraction or crestal bone loss resulting in loss of osseous height sufficient to place implants. Resorptive patterns in some patients along with sinus enlargement result in minimal bone that can accommodate implant placement. Maxillary sinus augmentation over the past 18 years with various bone graft materials has become routine treatment. Numerous studies have reported highly successful implant survival rates when placed into the augmented sinus. ${ }^{1-3}$ Transalveolar sinus floor elevation also referred to as subantrial augmentation was first described by Tatum $^{4}$ and later modified by Summers. ${ }^{5-7}$ This technique utilized a series of osteotomes with a mallet to create an osteotomy and subsequent infracturing of the sinus floor while elevating the Schneiderian membrane. Following manipulation, the space created in the sinus is augmented with various bone particulate graft materials increasing the volume of bone available for implant placement.

Various studies have reported that when $5 \mathrm{~mm}$ of residual alveolar bone is present, simultaneous implant placement can be preformed achieving adequate primary stability. ${ }^{6,89}$ But, when less than $5 \mathrm{~mm}$ of residual alveolar bone height is available, a delayed two-stage approach has been recommended. ${ }^{10,11}$ The most common complication of the lateral sinus elevation approach is typically tearing of the Schneiderian membrane which could allow for bacterial contamination or loose particles to gain access to the sinus cavity. A safer lateral window approach sinus augmentation procedure will be discussed using specialized safe cutting end drills with vertical stoppers for osseous window formation and subsequent membrane elevation (Lateral Approach Sinus Kit, Osstem/Hiossen).

\section{MATERIALS AND METHODS}

The lateral approach sinus kit (LAS Kit) (Osstem/Hiossen) provides 'Dome' drills, 'Core' drills, metal stoppers, side wall drill and a bone separator tool (Fig. 1).

The Dome drill is a unique osseous drill allowing removal of the lateral wall of the maxillary sinus while collecting autogenous bone to be added to the material to be placed into the sinus. Macro and micro cutting blades provide excellent cutting of the lateral wall without tearing of the sinus membrane. These Dome drills available in both 5.0 and $7.0 \mathrm{~mm}$ diameter are run at 1,200 to $1,500 \mathrm{RPM}$ with irrigation in an implant surgical handpiece. Metal depth control stoppers are provided that fit on the Dome drills limiting depth of penetration $(0.5,1.0,1.5,2.0,2.5$ and $3.0 \mathrm{~mm}$ ) and are used sequentially to safely expose the sinus membrane.

The Core drill, also available in 5.0 and $7.0 \mathrm{~mm}$ diameter, differs from the Dome drill in that the center does not cut, with bone removal resulting in a core of bone being left over the sinus. This boney lid may be elevated with the sinus membrane still attached becoming the new 'roof' to the sinus with osseous augmentation being placed below it. This particular drill follows the same design of the CAS kit (crestal augmentation sinus) drills and are utilized at 1,200 to 1,500 RPM. The metal drill stoppers also fit these drills allowing controlled sequential depth preparation. The bone separator tool is utilized to separate the osseous core created with the Core drill if removal is desired and is based on the practitioners preferred technique.

The side wall drill may be used to enlarge the osseous window created by the Dome drill if desired. The tip of this drill is smooth and designed to safely push the sinus membrane away from the cutting portion of the drill, which starts $1 \mathrm{~mm}$ from the safe end. Osseous cutting is performed 
at 1,500 RPM using the side of the rotating drill to enlarge the osseous window. The CAS kit metal drill stoppers may be placed on this drill to limit accidental penetration too far into the sinus and tearing of the membrane during this drills use. As with the other drills in this kit, irrigation is used during its use.

\section{CASE REPORT}

A male aged 32 presented with the desire for implant placement in the posterior maxillary right quadrant which had been missing the first molar for an extended period of time. The result of long-term loss of the tooth resulted in drifting of the second molar into the space which was corrected orthodontically prior to implant surgery. Radiographically, enlargement of the maxillary sinus was noted with insufficient height in the molar region for implant placement (Fig. 2). Resorption was noted compromising the width of the ridge at the buccal leading to a mild concavity (Fig. 3). Sinus augmentation was discussed to assist in achieving the patients desired treatment goal of implant placement and restoration with a fixed crown.

Following administration of local anesthetic, a crestal lingual incision was made with vertical releasing incisions at the mesial and distal aspect of the site, and a full thickness flap was elevated, leaving the attached gingiva undisturbed on the adjacent teeth (Fig. 4). Elevation of the flap extended superiorly to expose the lateral wall of the maxillary sinus up to the inferior aspect of the zygoma (Fig. 5).

A $5 \mathrm{~mm}$ wide Dome drill was placed on to the surgical handpiece with a $0.5 \mathrm{~mm}$ drill stopper (Fig. 6). This would allow initiation of the window without the possibility of excessive penetration and subsequent damage to the sinus membrane. The initial Dome drill is placed onto the surgical handpiece with the selected drill stop. The Dome drill with stopper was placed on the lateral sinus wall at a height more superior then the current height of the available bone as measured radiographically (Fig. 7). This is done to ensure that the window created has elevated the membrane circumferentially. When maximum depth has been achieved with the $0.5 \mathrm{~mm}$ drill stopper present, the drill stopper is changed to a $1.0 \mathrm{~mm}$ stopper and drilling is continued (Fig. 8). The drill stopper is sequentially increased checking for membrane exposure. Lateral drilling continues stepping up to the next drill stop (Fig. 9). Bone collected on the Dome drills is removed from the drill and placed into a sterile dish to be added to the graft to be placed, adding the hosts osteopotential cells to the graft (Fig. 10). As bone is removed over the sinus membrane, the area changes in color from

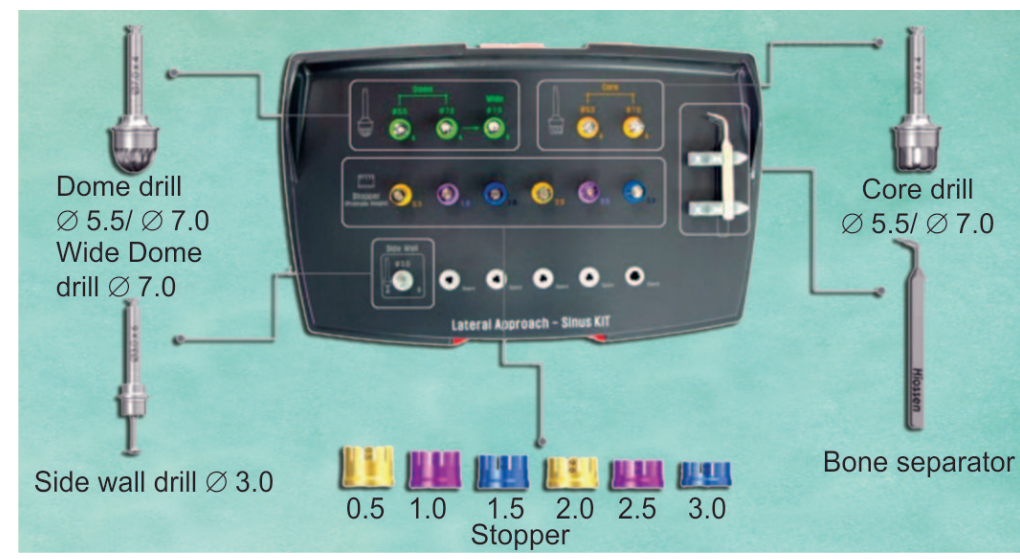

Fig. 1: Lateral approach sinus kit (LASK)
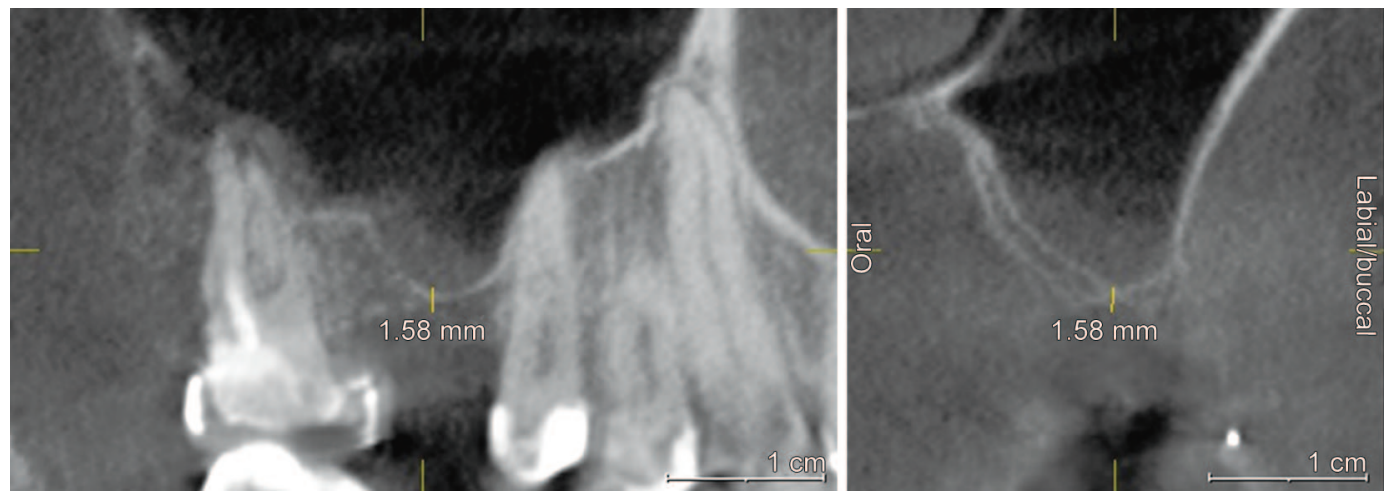

Fig. 2: $\mathrm{CBCT}$ radiograph pretreatment demonstrating insufficient osseous height for implant placement without sinus augmentation in the molar region 


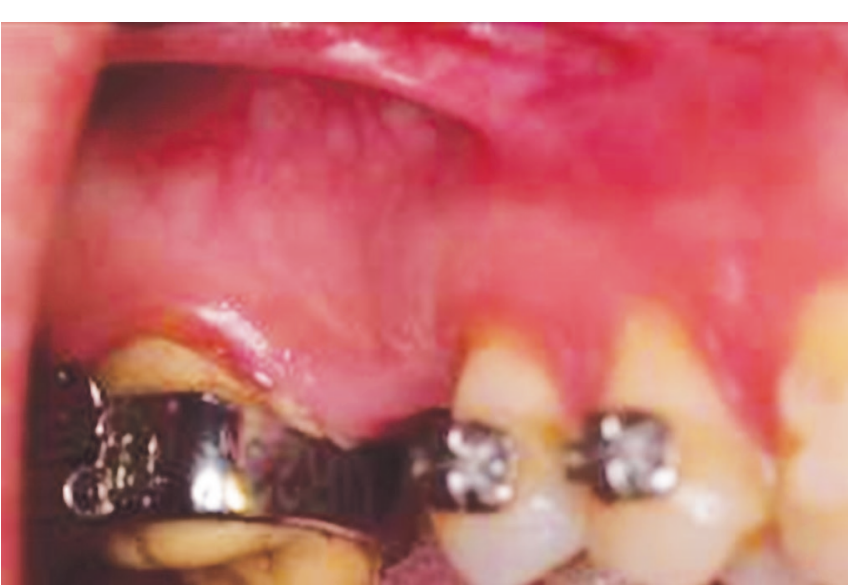

Fig. 3: Buccal concavity evident as a result of long standing loss of the first molar compromising the width of the site

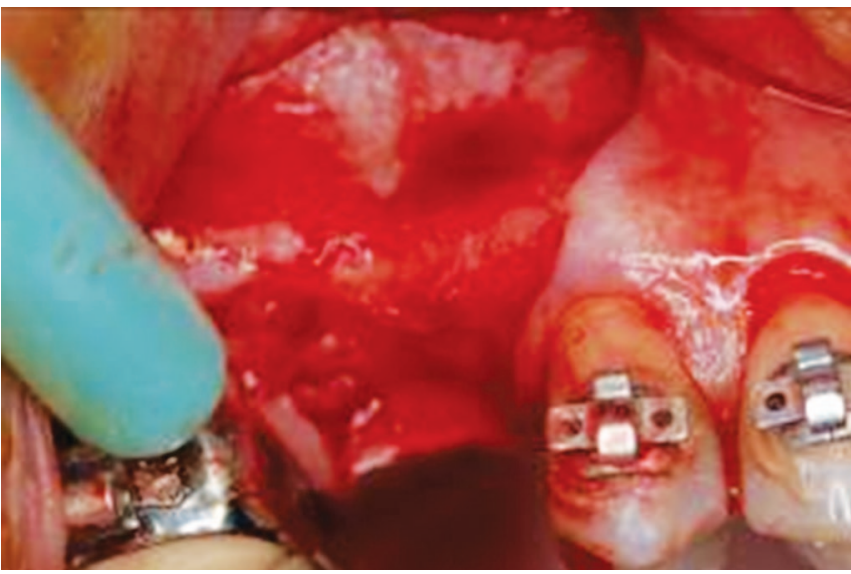

Fig. 5: Lateral aspect of the maxillary posterior following elevation of a full thickness flap

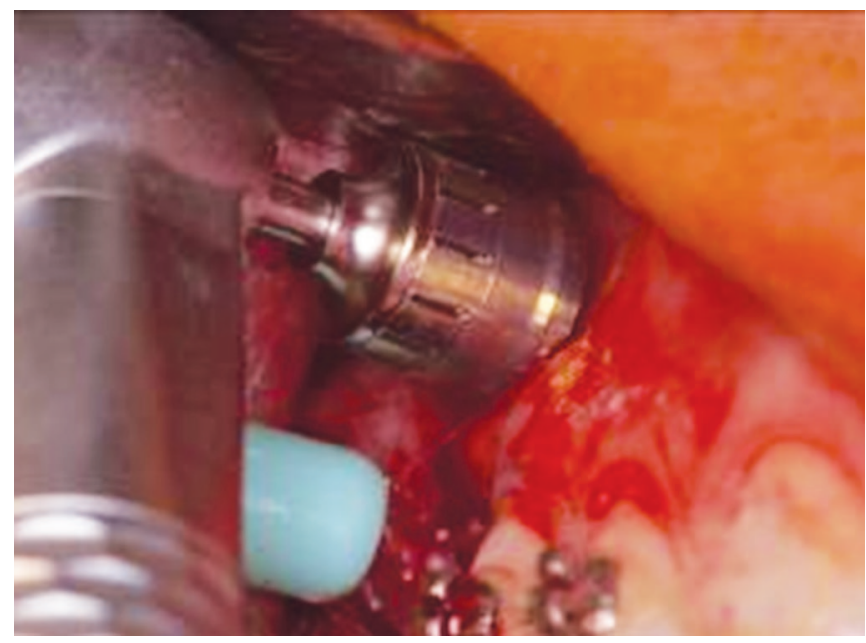

Fig. 7: Lateral sinus approached initiated with the Dome drill and a $0.5 \mathrm{~mm}$ drill stopper

the light color of the bone (ivory) to darker gray as the dark sinus begins to show clinically at the window (Fig. 11). As drilling is continued drill stops with increasing depth are used on the drill till full penetration of the lateral wall is achieved (Fig. 12).

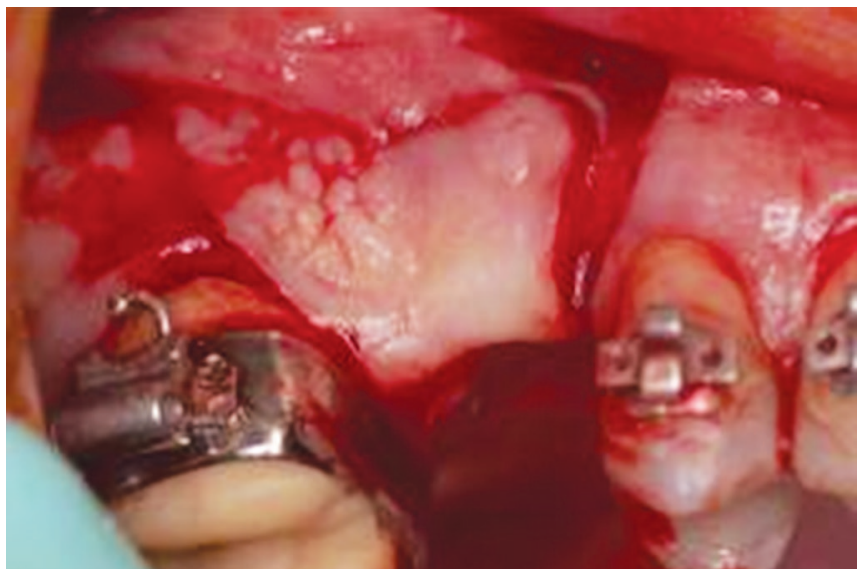

Fig. 4: A trapezoidal shaped flap was created with a scalpel with the crestal incision placed to the palatal aspect of the ridge

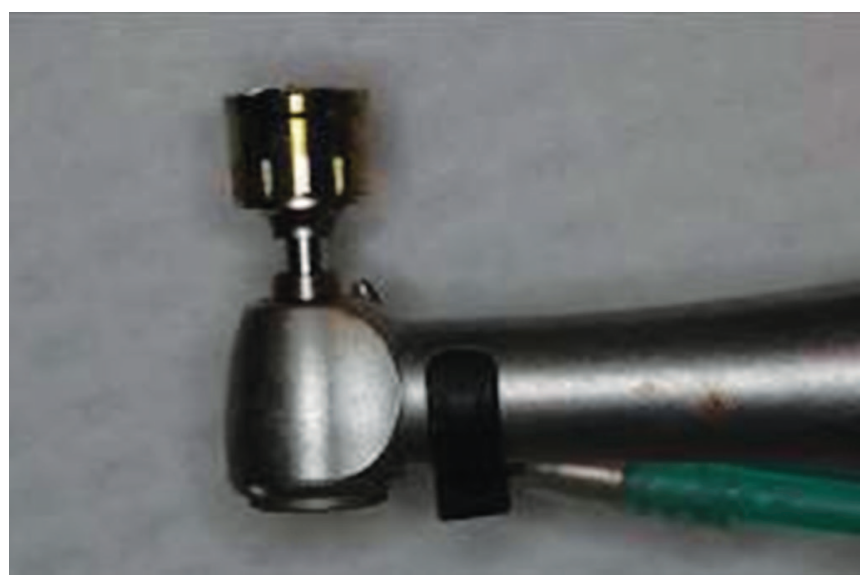

Fig. 6: Dome drill with $0.5 \mathrm{~mm}$ stopper placed on the surgical handpiece

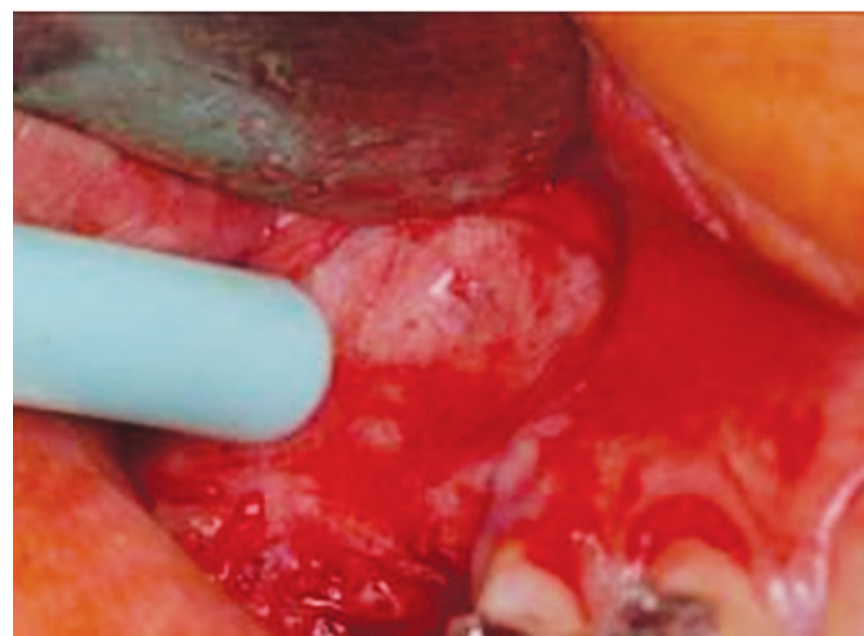

Fig. 8: The initial Dome drill created an outline into the bony wall

Final window creation is made with the Dome drill, in this particular case with a $2.5 \mathrm{~mm}$ drill stopper (Fig. 13). Some patients may require deeper drilling which is dependant on thickness of the lateral maxillary sinus wall. The intact sinus membrane is noted with no bone over the 


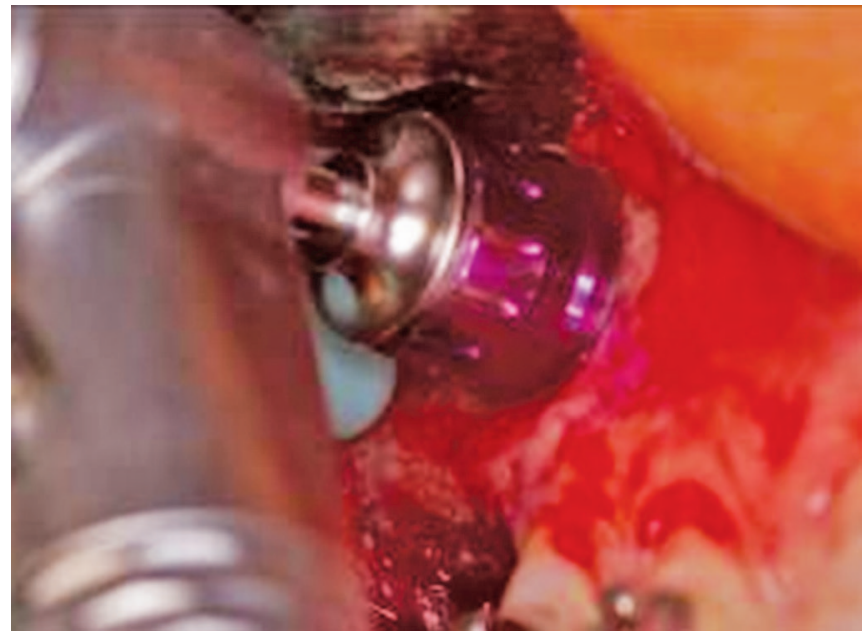

Fig. 9: Lateral sinus approached continued with the Dome drill and a $1.0 \mathrm{~mm}$ drill stopper

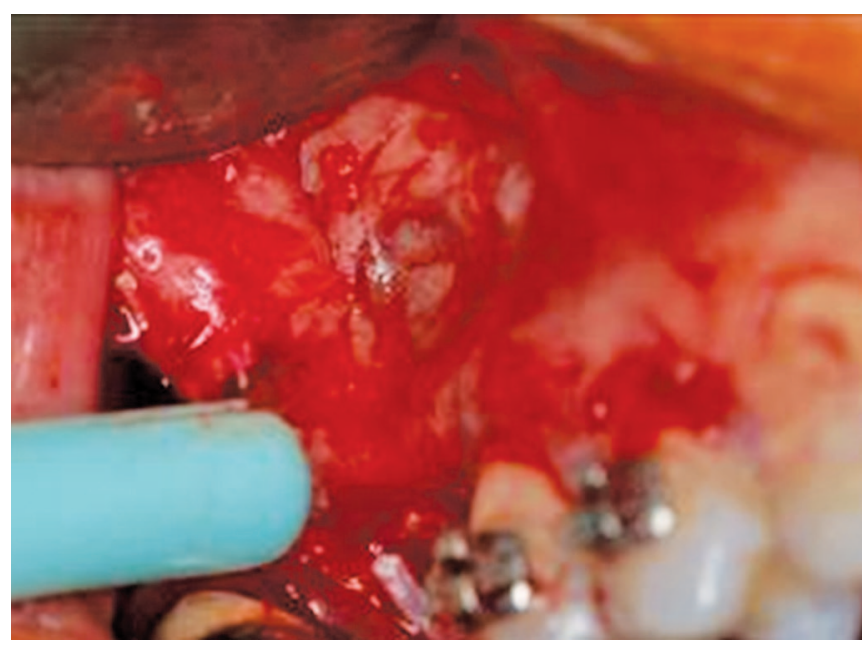

Fig. 11: Following each Dome drill, the site is examined for identification of the underlying membrane which will appear darker as bone is removed over it

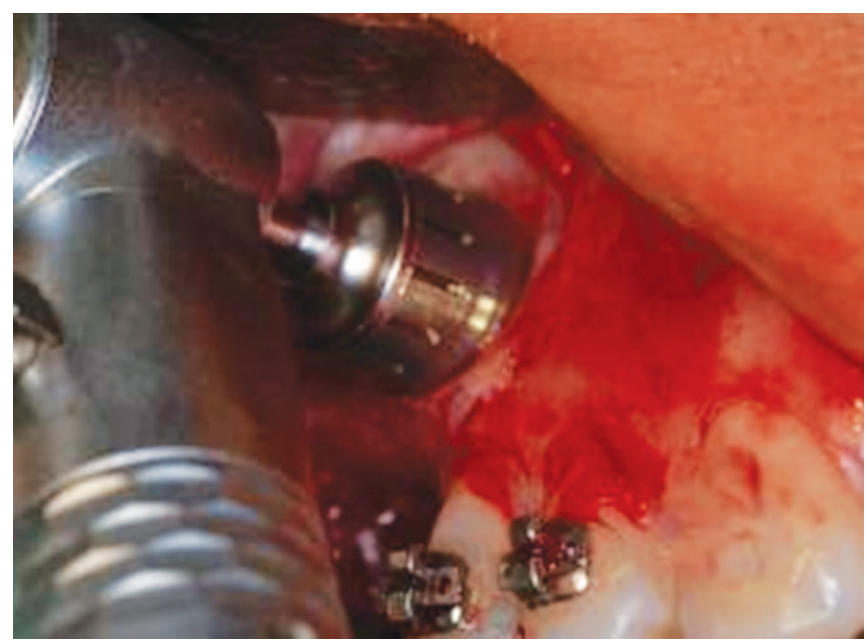

Fig. 13: Lateral sinus approached continued with the Dome drill and a $2.0 \mathrm{~mm}$ drill stopper

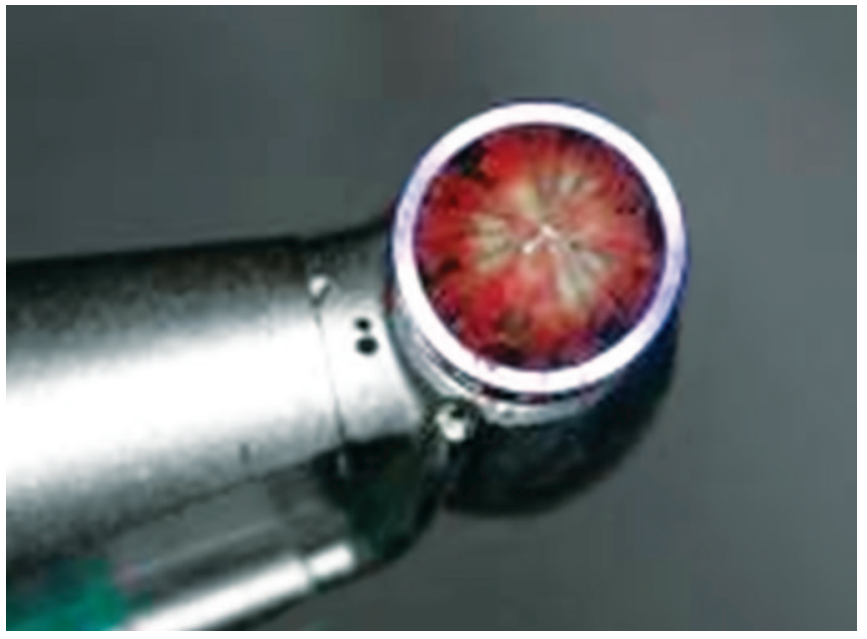

Fig. 10: Bone is collected from the Dome drill to be utilized to augment the graft to be placed

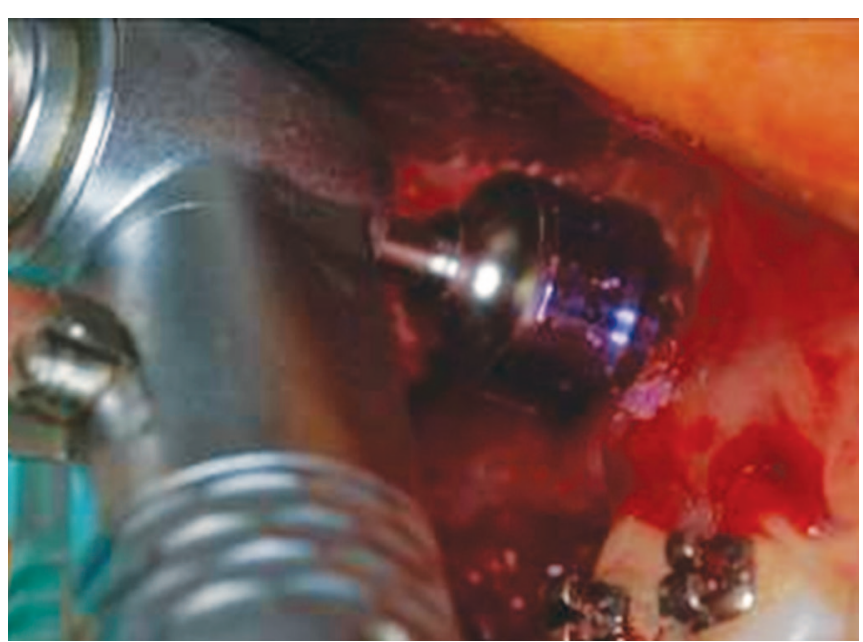

Fig. 12: Lateral sinus approached continued with the Dome drill and a $1.5 \mathrm{~mm}$ drill stopper

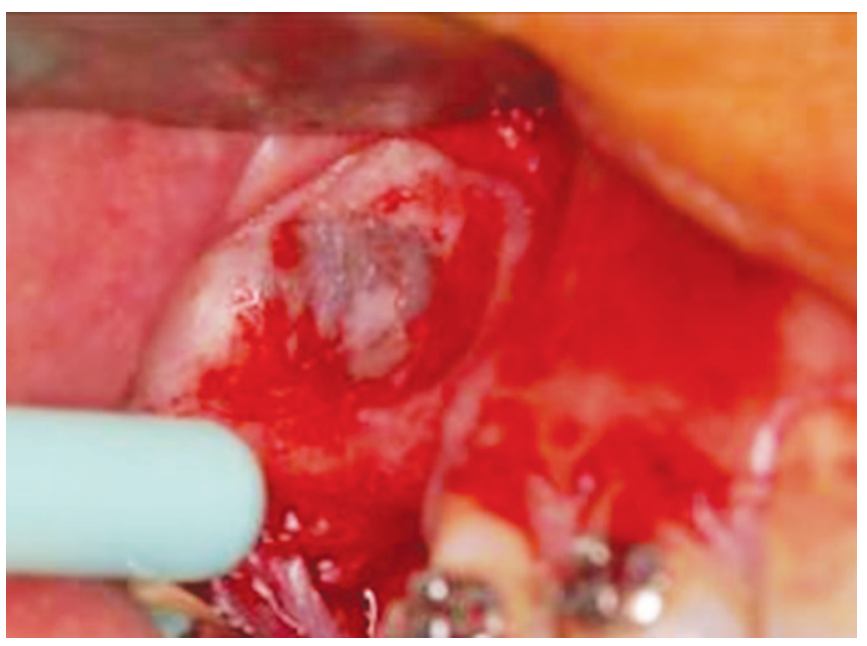

Fig. 14: Lateral wall of the maxillary sinus following sequential use of the Dome drill with increasing stopper depth demonstrating no damage to the sinus membrane after bone removal 
membrane at the window that has been created on the lateral wall (Fig. 14). Additionally, host bone is collected from the Dome drill.

Sinus curettes are utilized to start the sinus membrane elevation at the inferior aspect, teasing the membrane from the osseous wall of the sinus interiorly (Fig. 15). Following elevation of the membrane, the membrane should be intact and free of visible tears that may prevent graft distribution within the sinus during initial healing (Fig. 16). It is important that the elevation also include the medial wall of the sinus so that fills a volume great enough that the implant when placed will be surrounded by bone. Failure to elevate the medial aspect may result in the implant when placed having no osseous contact which may decrease clinical success following loading. Additionally, the authors advise elevation to a greater height then the implant length to be placed when a delayed fixture placement is to be performed. This will allow for possible graft settling during healing that may yield less height then was planned.

An absorbable extracellular membrane (Dynamatrix, Keystone Dental, Burlington, MA) is inserted into the sinus to act as protection containing the graft material and thicken the sinus membrane sealing any micro tears that might be present (Fig. 17). The resorbable membrane is cut to size and placed into the sinus dry using the patients blood in the site to wet it as its placed. Once wetted with blood, the resorbable membrane becomes sticky gluing itself to the sinus membrane.

Regenaform cortical cancellous bone chips (Exactech, Gainesville, FL) and sureOss, a freeze-dried cortical allograft (Osstem/Hiossen, Philadelphia, PA) in a 50:50 ratio in a sterile dappen dish and mixed with the autogenous bone collected from the Dome drill. The osseous graft mixture was carried to the oral cavity and introduced into the elevated sinus and gently condensed with a large plugger, pushing the mixture to the medial wall and filling in a lateral direction until the entire cavity was filled (Fig. 18). The process was repeated in the cavity anterior to the septa. Sufficient osseous graft was placed till the sinus was augmented to be flush with the outer aspect of the lateral sinus wall at the window that had been created (Fig. 19).

Following sinus grafting, the site was prepared and an implant (4.5 × $10 \mathrm{~mm}$, ETIII, Osstem/Hiossen, Philadelphia, PA) was placed and the site. A low profile cover screw was used to allow primary closure of the flap. The radiograph shows initial graft placement and the elevation achieving a site that can accommodate implant placement at this surgical appointment (Fig. 20).

A long-term resorbable membrane (DynaMatrix) was cut to extend beyond the outline of the lateral window and placed over the osseous graft that had been placed into the sinus (Fig. 21). The flap was repositioned and initially closed

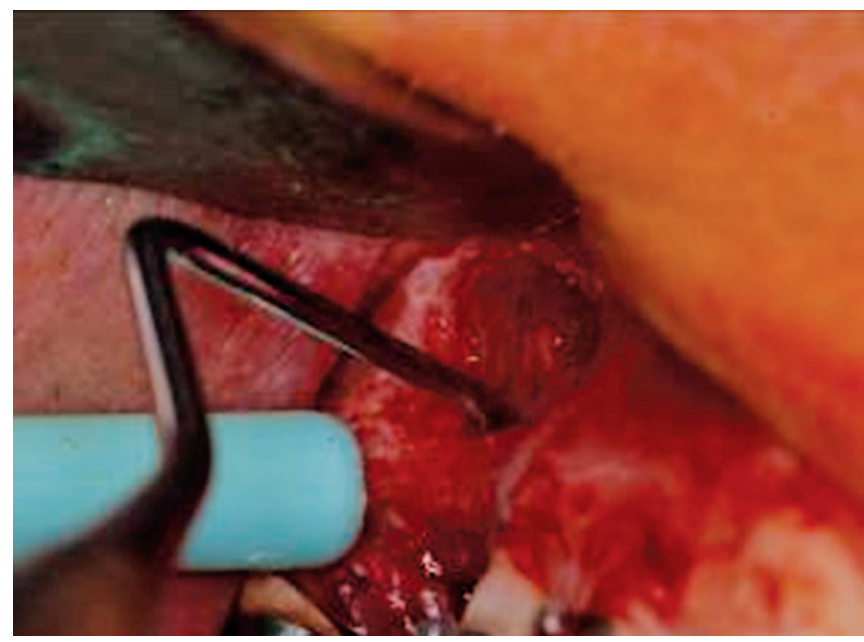

Fig. 15: A curette is utilized to separate the sinus membrane from the bone of the maxillary sinus, elevating it superiorly from the inferior floor to the medial wall

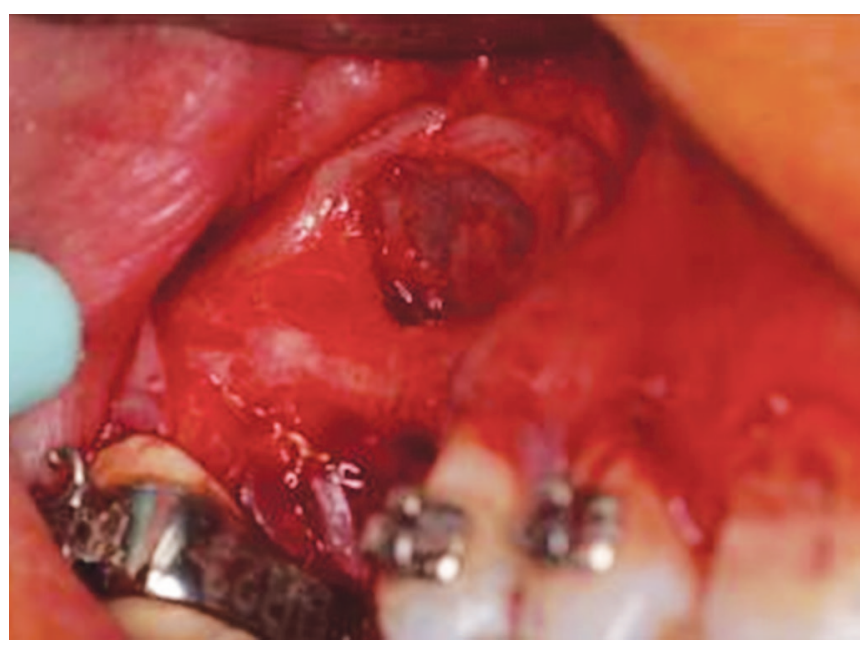

Fig. 16: The lateral window is completed, demonstrating an intact sinus membrane following the use of the Dome drills with stoppers

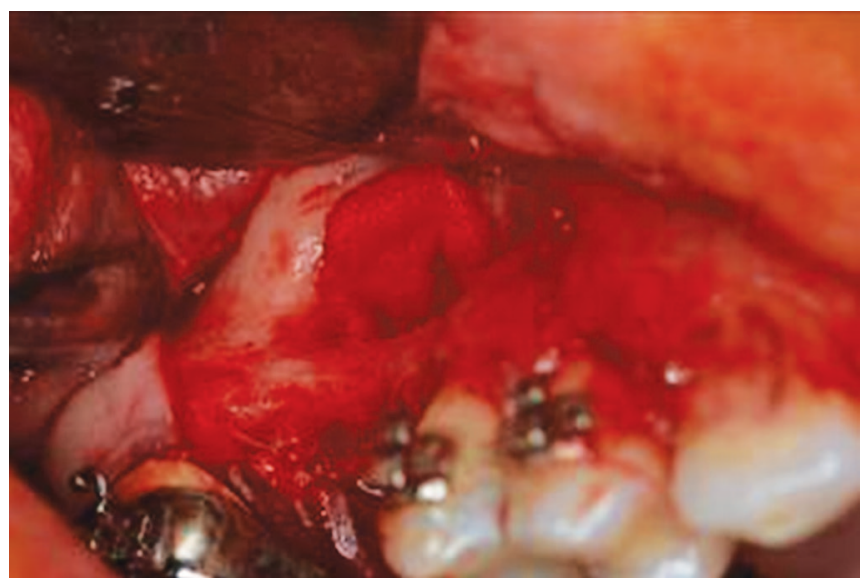

Fig. 17: A collagen membrane is placed into the sinus overlying the elevated sinus membrane, to help confine the graft material being placed should a micro tear be present in the elevated sinus membrane

with a horizontal mattress suture using a 5-0 cytoplast suture material (Osteogenics Biomedical, Inc., Lubbock, TX) to achieve primary closure of the flap without tension then 


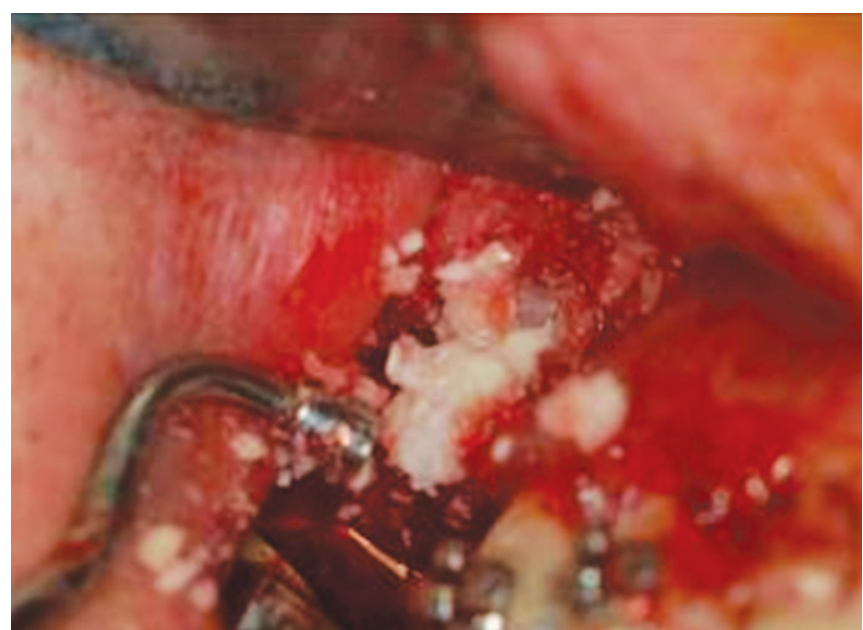

Fig. 18: Osseous graft material was mixed with the patients donor bone collected from the Dome drills and is gently packed into the sinus

the crest was closed with interrupted sutures (Fig. 22). This suture serves to resist soft tissue tension that may result due to inflammation and the resulting swelling following surgery. Additional sutures are placed to close the incision line using a simple interrupted technique.

The patient returned 8 months following implant placement. Soft tissue in the site on the lateral aspect demonstrated no inflammation and incision lines were not discernible on the gingiva. The implant was exposed using a disposable tissue punch and the cover screw was replaced by a healing abutment. A radiograph was taken to check and verify the organization of the osseous graft that had been placed into the sinus, integration of the implant and seating of the healing abutment on the fixture (Fig. 23). A CBCT was taken to check the graft and implant integration and the implant is ready to be restored (Fig. 24).

\section{CONCLUSION}

Emphasis has moved to the use of a crestal approach to sinus elevation when additional osseous height is required for implant placement. This approach works well when at least $5 \mathrm{~mm}$ of osseous height is present for immediate implant placement. Yet, when less bone height is present, a lateral window approach may be the preferred technique to increase crestal height and geometric volume so that implant fixtures may be placed.

The lateral sinus augmentation approach can be challenging as tearing of the sinus membrane often necessitates abandoning the procedure and re-entry at a later date after the membrane has healed. Previous techniques involved use of diamonds or carbides in a highspeed handpiece or the use of Piezo surgical units. These approaches had potential for membrane damage (burs in a highspeed) or were very slow (Piezo). The LAS kit, from

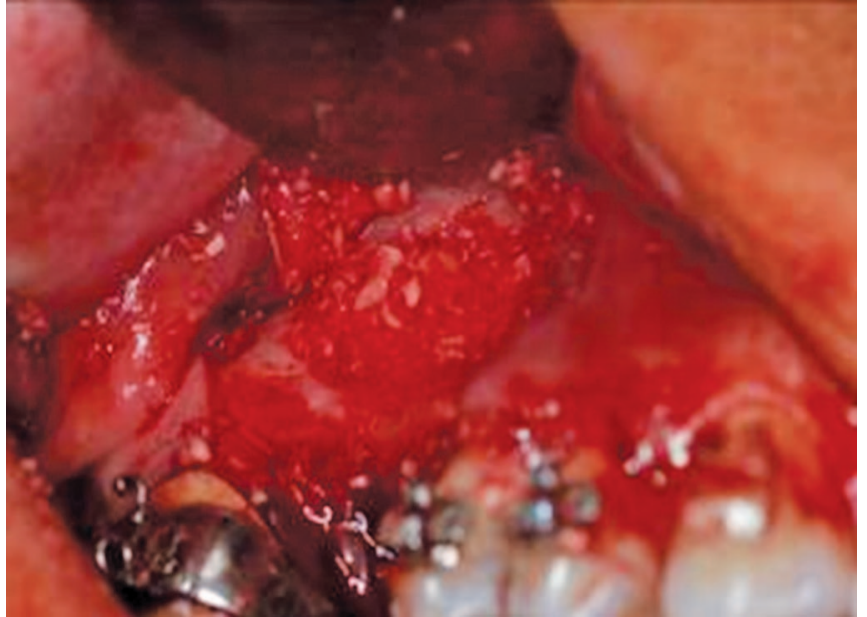

Fig. 19: The elevated sinus area has been completely packed with osseous graft material

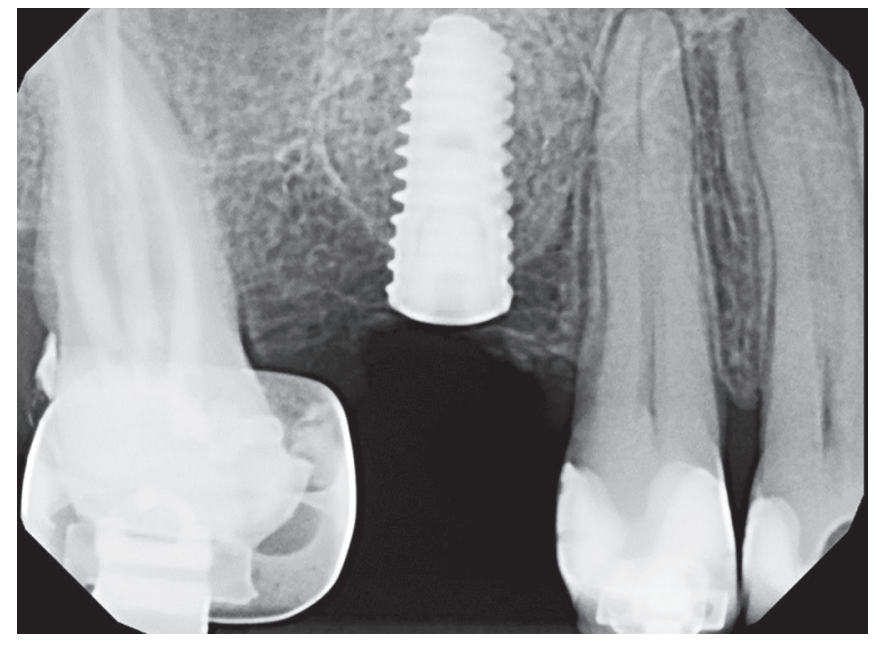

Fig. 20: Implant placement following osseous graft healing demonstrating the new sinus height achieved

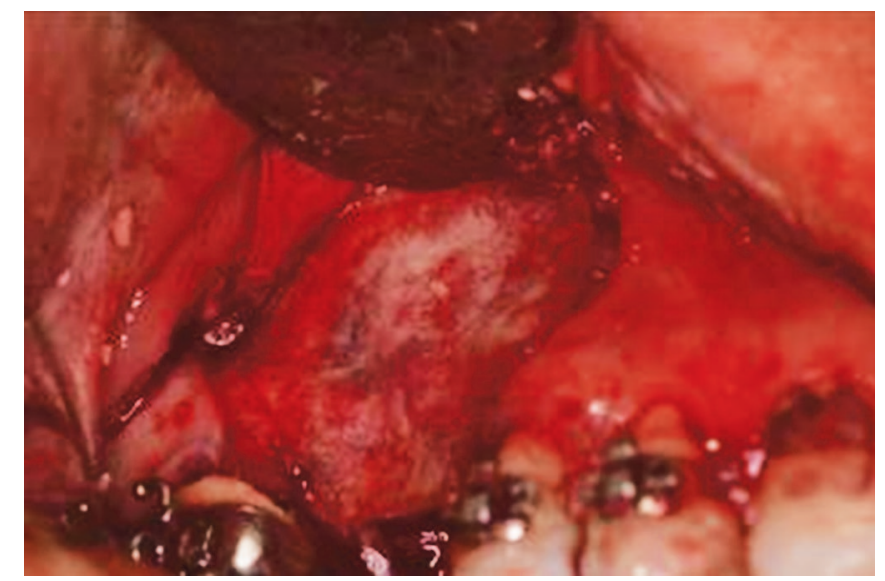

Fig. 21: A resorbable membrane was placed over the bony sinus window to limit soft tissue ingrowth into the graft during the healing phase

Osstem/Hiossen, utilizes special designed drills that greatly minimize tearing of the membrane and improve the safety of the procedure. 


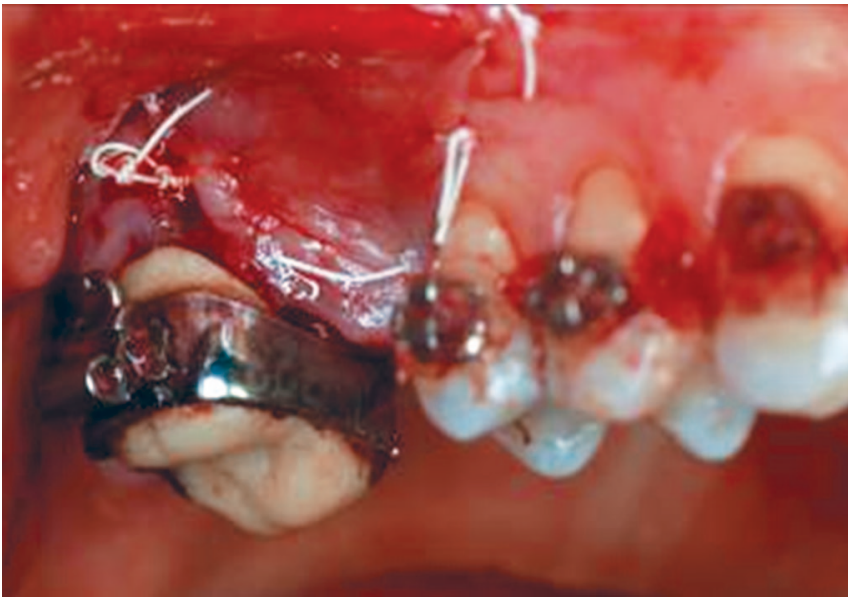

Fig. 22: The flap was repositioned and closed with a horizontal mattress and interrupted sutures

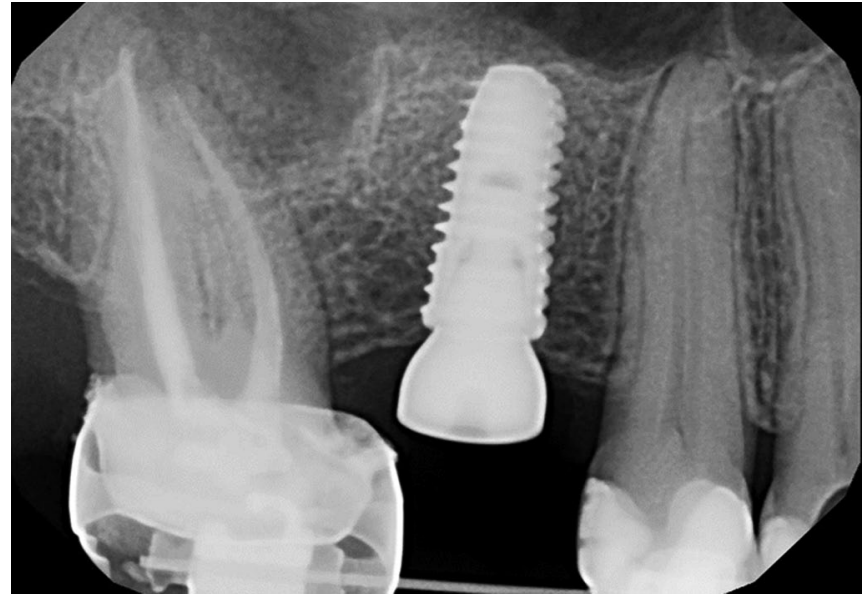

Fig. 23: Implant following 8 months healing and exposure to place a healing abutment demonstrating blending of the grafted sinus with the surrounding native bone
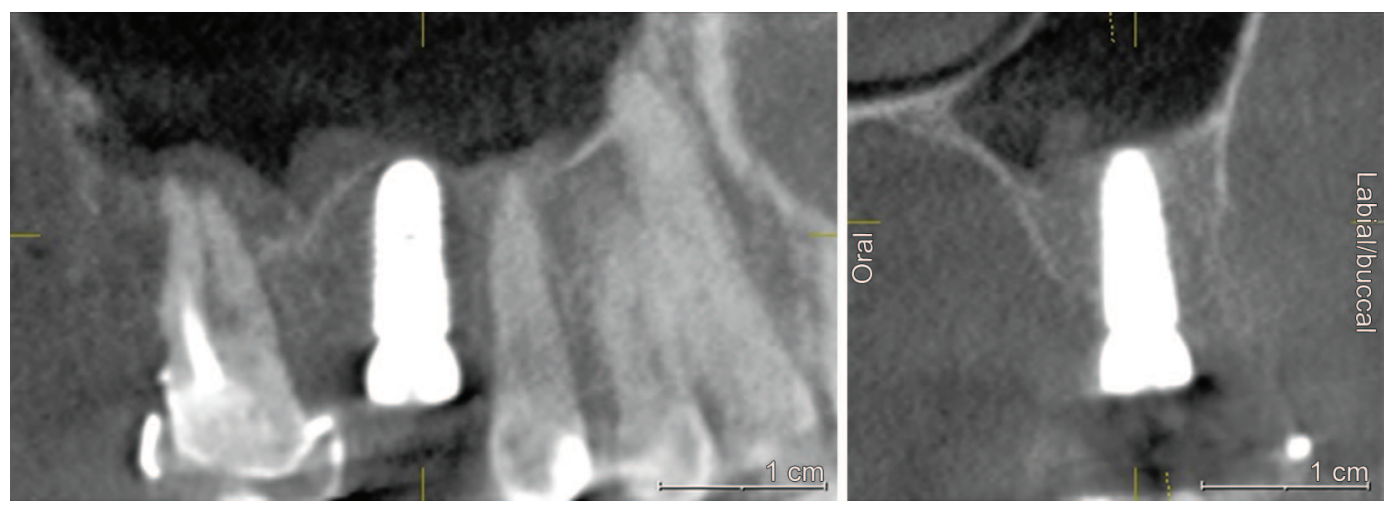

Fig. 24: CBCT demonstrating new volume of bone achieved following sinus augmentation and implant placement which is ready for restoration of the implant

\section{REFERENCES}

1. Blomqvist JE, Alberius P, Isaksson S. Two maxillary sinus reconstruction with endosseous implants: A prospective study. Int J Oral Maxillofac Implants 1998;13:758-766.

2. Valentini P, Abensur DJ. Maxillary sinus grafting with an organic bovine bone: A clinical report of long-term results. Int J Oral Maxillofac Implants 2003;18:556-560.

3. Tong DC, Drangsholt M, Beirne OR. A review of survival rates for implants placed in grafted maxillary sinuses using meta-analysis. Int J Oral Maxillofac Implants 1998;13:175-182.

4. Tatum OH Jr. Maxillary and sinus implant reconstructions. Dent Clin North Am 1986;30:207-229.

5. Rosen PS, Summers R, Mellado Jr, et al. The bone-added osteotome sinus floor elevation technique: multicenter retrospective report of consecutively treated patients. Int J Oral Maxillofac Implants 1999;14:853-858.

6. Summers RB. A new concept in maxillary implant surgery: the osteotome technique. Compend Contin Educ Dent 1994;15: 152-162.

7. Summers RB. The osteotome technique: part 3-less invasive methods of elevating the sinus floor. Compend Contin Educ Dent 1994;15:698-710.

8. Emmerich D, Att W, Stappert C. Sinus floor elevation using osteotomes: a systemic review and meta-analysis. J Periodontal 2005;76:1237-1251
9. Tofflen M. Osteotome-mediated sinus floor elevation: a clinical report. Int J Oral Maxillofac implants 2004;19:266-273.

10. Peleg M, Mazor Z, Chaushu G, Garg AK. Sinus floor augmentation with simultaneous implant placement in the severely atrophic maxilla. J Periodontal 1998;69:1397-1403.

11. Peleg M, Mazor Z, Garg AK. Augmentation grafting of the maxillary sinus and simultaneous implant placement in patients with 3 to $5 \mathrm{~mm}$ of residual alveolar bone height. Int J Oral Maxillofac Implants 1999;14:549-556.

\section{ABOUT THE AUTHORS}

\section{Gregori M Kurtzman}

Private General Practice, Silver Spring, Maryland, USA

Correspondence Address: General Practitioner, Leisure World Plaza Professional Building, 3801 International Drive, Suite 102, Silver Spring, MD 20906, USA, Phone: 301-5983500, Fax: 301-5989046 e-mail: dr_kurtzman@maryland-implants.com

\section{Douglas F Dompkowski}

Private Practice, Department of Periodontology and Implant Dentistry Bethesda, Maryland; Faculty, Department of Periodontology University of Maryland, Maryland, USA 